\title{
Diagnosis of Brain Lesions, Glioma, Multiple-Sclerosis and Metastases from MRI: An efficient classifier-aided method using Refractive Index as a surrogate Biological Marker
}

\author{
Sparsh Jain, ${ }^{1,2}$ Tapan K Biswas, ${ }^{1}$ Rajib Bandyopadhyay \\ ${ }^{1}$ Department of Instrumentation and Electronics Engineering, Jadavpur University, Kolkata, India; \\ ${ }^{2}$ (Former) Harvard University, Brigham and Women's Hospital, Boston MA, United States of America; \\ sparshju@gmail.com
}

\begin{abstract}
We introduce a highly accurate method of diagnosing the various pathological conditions that might exist in a subject's brain, like edema, multiple sclerosis (Tumefactive, Relapsing-remitting, secondaryprogressive, primary-progressive and progressive-relapsing), glioma, glioblastoma and metastases. These show up on conventional MRI scans, but it is often difficult to identify the exact type of the pathology from the grayscale image. We employ the use of Support Vector Machines (SVM) to work on the MR Spectroscopy $[6,12]$ data and correctly identify the condition-especially in seemingly vague cases where radiologists cannot rule out high uncertainty in their conclusion. The SVM trains on data sets collected for different patients and optimizes its hyperplanes based on eight input variables - T2, $\mathrm{CHO}, \mathrm{ADC}, \mathrm{CR}, \mathrm{CHO} / \mathrm{NAA}, \mathrm{CR} / \mathrm{NAA}, \mathrm{LIP} / \mathrm{LAC}, \mathrm{MI}, \mathrm{CH} / \mathrm{CR}, \mathrm{T} 2$ periphery [6] and Refractive index. Refractive index is an additional parameter which we include to get better boundary lines and accuracy, as shown in our prior works [10]. We test this SVM on a set of 19 patients' data and achieve $100 \%$ accuracy in predictions. The training and testing is carried out in MATLAB.
\end{abstract}

Keywords: Magnetic Resonance Imaging, MR Spectroscopy, Refractive Index, Support Vector Machine (SVM), Brain Lesions, Cancer.

\section{Introduction}

Only structural changes of MR images of aneurysms, Glioma or other brain tumours including metastasis or secondary deposits of cancer tissues cannot be sufficient for accurate diagnosis [1]. Data collected from metabolites of MR spectroscopy like NAA, Choline, Creatine, lipid or lactate or physical data like Refractive Indices (RI), T2 magnetic relaxation values, and Apparent Diffusion Coefficient (ADC) values are also important for correct diagnosis of the disease. Figures 1 to 4 show how a few of these diseases appear on the scans [Fig 1-4].

If the supporting data are available, live prediction of diseases or of the tissue can be performed with 90 to $95 \%$ accuracy using Support Vector Machines (SVM). Clustering of diseases and tissues can also be done using SVMs. It is worth mentioning that machine learning tools like clustering, SVM and neural 
networks (Principal Component Analysis) have previously been used for similar purposes on MRI data [5, 10, 13-15].

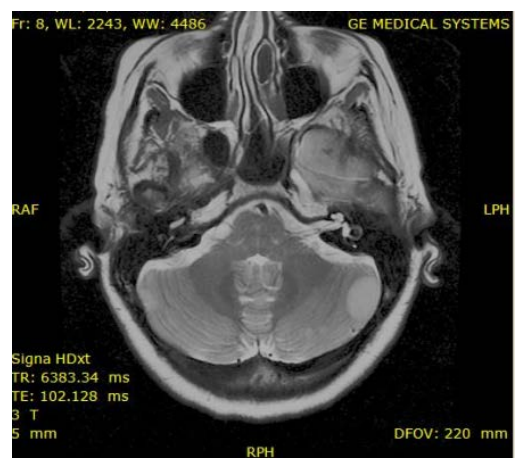

Figure 1: Metastasis MRI

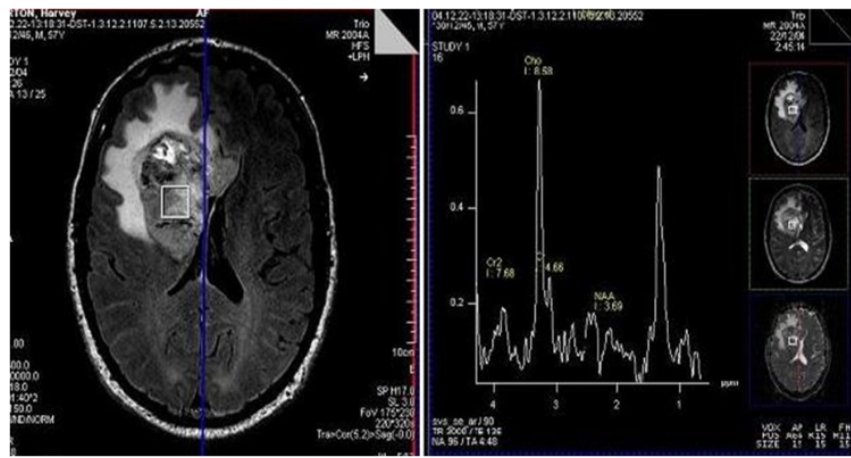

Figure 3: Grade-3 Glioblastoma with abnormal MRS

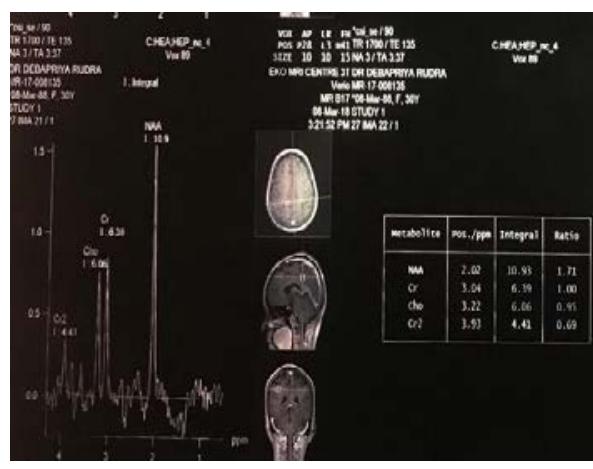

Figure 2: MR Spectroscopy (MRS) Normal

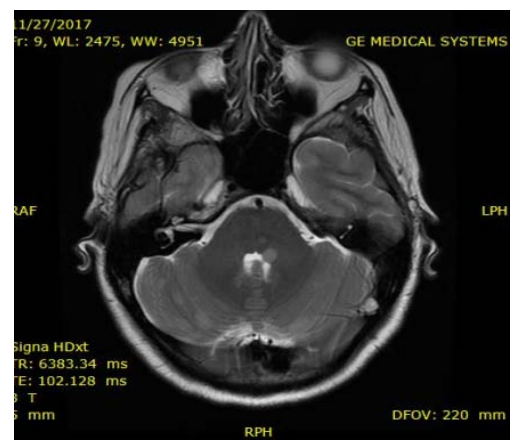

Figure 4: Multiple Sclerosis (MS) MRI

\section{Background}

SVM employs a nonlinear classification method [2]. It is implemented to assess and make virtual pathological predictions using the data obtained from MRI, various metabolic components and their ratio of MR Spectroscopy, ADC, RI and T2 values (Table 1 ).

An SVM with extraordinary data processing uniqueness, nonlinearity and learning with generalization capability is used to characterize the disease. Thus there are 10 independent numeric variables. SVM and Neural Network (NN) both belong to supervised learning methods, but their working procedure is different.

We have used Error correcting output codes (ECOC) mode to reduce the errors in classification. ECOC is an error-correcting output codes classifier used to get multiclass learning by diminishing multiple binary classifiers for instance SVMs [3].

The main factor responsible for the performance of ECOC methods is the self-determination of binary classifiers, otherwise the ECOC method will be unsuccessful. It is effective in multiple classes and requires a coding design. This design determines the classes. Binary learners undergo training and a decoding system determines the prediction of the binary classes.

a) The coding design is one-versus-one. 
Sparsh Jain, Tapan K Biswas, Rajib Bandyopadhyay; Diagnosis of Brain Lesions, Glioma, Multiple-Sclerosis and Metastases from MRI: An efficient classifier-aided method using Refractive Index as a surrogate Biological Marker. Journal of Biomedical Engineering and Medical Imaging, Volume 5, No 3, June (2018), pp -19-26

b) Decoding procedure would utilize loss $g$.

c) SVMs will be the learners.

SVM utilizes a function or hyperplane [4] among different classes giving a maximum margin parameter. This hyperplane tries to divide the classes so that each groups remains on either side of the plane and by a particular boundary. Our SVM handles 10 variables. It has 8 output results or classes of different types of tissue (such as CSF, gray matter, white matter) and diseases like low and high grade glioma and metastasis for targeted prediction.

Improved classification accuracy can be achieved by ECOC models compared to other multiclass model.

\section{Method}

The data has been used from the authors' prior research [5] (Table1). We train the SVM using data from 116 patients. The SVM is then tested on data from 19 patients.

The method consists of two parts:

a) Training the SVM using available data

b) Testing the trained SVM to classify unknown data.

\subsection{Source Code and Platform}

The SVM training and classification work has been implemented on the 64-bit MATLAB R2017a environment on Windows 10 Home platform. Access to the source code and complete training data can be requested by contacting the corresponding author.

\subsection{Training the Support Vector Machine}

\subsection{Identification}

First we identify the label and data. Label includes the names of tissue or diseases like MS, Glioma etc. Data corresponds to all the numerical values associated with each label. SVM essentially matches the data sets with the correct labels. The first column of Table 1 has all the labels. The remaining 11 columns contain the data. So for example, row no. 5 has the label CSF and that particular CSF scan has 11 values corresponding to $\mathrm{T} 2, \mathrm{ADC}, \mathrm{CR}, \mathrm{CHO}$ etc [6].

\section{Keys for tables 1-4:}

'CSF': Cerebrospinal fluid

'gmatter': Gray Matter

'gblastma': Glioblastoma
'MS': Multiple sclerosis

'w matter': White Matter

'mets': Metastases 
Journal of Biomedical Engineering and Medical Imaging, Volume 5, No 3, June 2018

Table 1: Preview of the entire data set. 41 out of 135 sets are shown below.

\begin{tabular}{|c|c|c|c|c|c|c|c|c|c|c|c|}
\hline TISSUE & $\mathrm{T} 2$ & $\mathrm{CHO}$ & $A D C$ & CR & $\mathrm{CHO} / \mathrm{NAA}$ & CR/NAA & LIP/LAC & $\mathrm{MI}$ & $\mathrm{CH} / \mathrm{CR}$ & T2peri & $\mathrm{RI}$ \\
\hline CSF & 400 & 1610 & 300 & 1400 & 0.402 & 0.346 & 1400 & 910 & 1.15 & 400 & 1.3333 \\
\hline CSF & 399 & 1676 & 307 & 1450 & 0.404 & 0.347 & 1489 & 917 & 1.15 & 399 & 1.3333 \\
\hline CSF & 398 & 1689 & 311 & 1560 & 0.408 & 0.351 & 1550 & 957 & 1.15 & 399 & 1.3333 \\
\hline CSF & 397 & 1700 & 313 & 1600 & 0.409 & 0.357 & 1554 & 987 & 1.15 & 399 & 1.3333 \\
\hline CSF & 396 & 1728 & 320 & 1788 & 0.412 & 0.361 & 1660 & 1050 & 1.14 & 395 & 1.3333 \\
\hline CSF & 395 & 1711 & 322 & 1800 & 0.422 & 0.367 & 1701 & 1056 & 1.14 & 395 & 1.3333 \\
\hline CSF & 394 & 1710 & 322 & 1809 & 0.423 & 0.368 & 1690 & 1059 & 1.14 & 394 & 1.3333 \\
\hline CSF & 345 & 2021 & 402 & 2060 & 0.572 & 0.448 & 1744 & 1145 & 1.15 & 345 & 1.3333 \\
\hline CSF & 344 & 2022 & 403 & 2061 & 0.573 & 0.451 & 1744 & 1145 & 1.15 & 344 & 1.3333 \\
\hline CSF & 343 & 2023 & 404 & 2062 & 0.574 & 0.452 & 1745 & 1146 & 1.15 & 343 & 1.3333 \\
\hline CSF & 342 & 2024 & 405 & 2068 & 0.577 & 0.453 & 1746 & 1147 & 1.15 & 342 & 1.3333 \\
\hline CSF & 341 & 2123 & 411 & 2063 & 0.578 & 0.453 & 1747 & 1148 & 1.15 & 341 & 1.3333 \\
\hline $\mathrm{ms}$ & 340 & 11750 & 145 & 8320 & 0.779 & 0.557 & 4160 & 2912 & 1.4 & 340 & 1.3334 \\
\hline $\mathrm{ms}$ & 339 & 11750 & 1460 & 8319 & 0.778 & 0.541 & 4423 & 3223 & 1.4 & 339 & 1.3335 \\
\hline $\mathrm{ms}$ & 338 & 11749 & 1459 & 8314 & 0.776 & 0.538 & 4423 & 3221 & 1.4 & 338 & 1.3336 \\
\hline $\mathrm{ms}$ & 337 & 11746 & 1445 & 8311 & 0.774 & 0.536 & 4421 & 3220 & 1.4 & 337 & 1.3421 \\
\hline $\mathrm{ms}$ & 336 & 11745 & 1444 & 8310 & 0.773 & 0.534 & 4422 & 3219 & 1.4 & 336 & 1.3439 \\
\hline $\mathrm{ms}$ & 335 & 11745 & 1443 & 8309 & 0.772 & 0.532 & 4420 & 3216 & 1.4 & 335 & 1.3498 \\
\hline $\mathrm{ms}$ & 334 & 11743 & 1443 & 8308 & 0.771 & 0.531 & 4419 & 3214 & 1.4 & 334 & 1.3499 \\
\hline $\mathrm{ms}$ & 304 & 5947 & 120 & 5400 & 0.873 & 0.7396 & 6766 & 4294 & 1.1 & 245 & 1.3519 \\
\hline $\mathrm{ms}$ & 249 & 3448 & 112 & 3320 & 0.821 & 0.7112 & 5423 & 2322 & 1.02 & 230 & 1.3589 \\
\hline $\mathrm{ms}$ & 245 & 1610 & 110 & 2212 & 0.465 & 0.941 & 1440 & 2276 & 0.495 & 227 & 1.3641 \\
\hline gmatter & 130 & 1601 & 72 & 2209 & 0.464 & 0.938 & 1439 & 361 & 0.491 & 166 & 1.3956 \\
\hline gmatter & 129 & 1599 & 73 & 2208 & 0.463 & 0.936 & 1437 & 357 & 0.4911 & 165 & 1.3956 \\
\hline gmatter & 128 & 1597 & 74 & 2206 & 0.463 & 0.934 & 1435 & 351 & 0.489 & 165 & 1.3956 \\
\hline w matter & 95 & 1180 & 70 & 2443 & 0.453 & 0.788 & 1345 & 312 & 0.488 & 148 & 1.4251 \\
\hline w matter & 93 & 1108 & 71 & 2435 & 0.447 & 0.771 & 1341 & 320 & 0.468 & 146 & 1.4256 \\
\hline w matter & 93 & 1067 & 89 & 2301 & 0.444 & 0.775 & 1167 & 324 & 0.466 & 169 & 1.4262 \\
\hline edema & 160 & 1231 & 132 & 2216 & 0.443 & 0.776 & 1123 & 325 & 0.467 & 246 & 1.3741 \\
\hline edema & 182 & 1331 & 130 & 2321 & 0.442 & 0.787 & 1011 & 321 & 0.456 & 243 & 1.3823 \\
\hline edema & 191 & 1451 & 131 & 2356 & 0.441 & 0.778 & 990 & 313 & 0.445 & 245 & 1.3822 \\
\hline edema & 193 & 1452 & 130 & 2340 & 0.441 & 0.768 & 990 & 312 & 0.445 & 245 & 1.3823 \\
\hline GLIOMA & 90 & 1443 & 127 & 2243 & 0.431 & 0.766 & 989 & 310 & 0.423 & 175 & 1.4331 \\
\hline GLIOMA & 99 & 1365 & 177 & 2254 & 0.341 & 0.712 & 917 & 300 & 0.343 & 170 & 1.4339 \\
\hline GLIOMA & 107 & 2213 & 155 & 2114 & 0.333 & 0.677 & 901 & 310 & 0.321 & 191 & 1.4456 \\
\hline Gblastma & 108 & 2457 & 154 & 2115 & 0.332 & 0.676 & 900 & 311 & 0.311 & 195 & 1.4512 \\
\hline Gblastma & 109 & 2655 & 152 & 2112 & 0.332 & 0.676 & 900 & 311 & 0.311 & 195 & 1.4539 \\
\hline Gblastma & 128 & 1284 & 131 & 2589 & 0.541 & 0.781 & 1767 & 322 & 0.76651 & 198 & 1.4723 \\
\hline METS & 129 & 1298 & 130 & 2567 & 0.511 & 0.657 & 1011 & 323 & 0.432 & 200 & 1.4831 \\
\hline METS & 130 & 1301 & 130 & 2478 & 0.511 & 0.657 & 1011 & 323 & 0.432 & 200 & 1.4831 \\
\hline METS & 152 & 1412 & 132 & 2022 & 0.425 & 0.713 & 1121 & 357 & 0.451 & 224 & 1.4913 \\
\hline
\end{tabular}


Sparsh Jain, Tapan K Biswas, Rajib Bandyopadhyay; Diagnosis of Brain Lesions, Glioma, Multiple-Sclerosis and Metastases from MRI: An efficient classifier-aided method using Refractive Index as a surrogate Biological Marker. Journal of Biomedical Engineering and Medical Imaging, Volume 5, No 3, June (2018), pp -19-26

\subsubsection{Training}

We use the Fitcecoc command in Matlab to train an SVM for the data and label we already have (Table 2). SVM uses Supervised Learning and classifies data. The basic command for SVM training is FitSVM or svmtrain but that can be used only for binary classification i.e. when there are only two classes/labels [7]. In this case we have 8 different labels, so we use fitcecoc, which can accommodate multiple classes. SVM and Neural networks both work on supervised learning but their mechanisms differ.

So the output variable generated is the Support Vector Machine trained using the data.

Table 2: Preview of the Training data set. 16 out of 116 sets are shown below.

\begin{tabular}{|l|r|r|r|r|r|r|r|r|r|r|r|}
\hline TISSUE & \multicolumn{1}{|c|}{ T2 } & \multicolumn{1}{c|}{ CHO } & \multicolumn{1}{|c|}{ ADC } & \multicolumn{1}{c|}{ CR } & CHO/NAA & CR/NAA & LIP/LAC & MI & \multicolumn{1}{l|}{ CH/CR } & T2peri & RI \\
\hline & & & & & & & & & & & \\
\hline CSF & 400 & 1610 & 300 & 1400 & 0.402 & 0.346 & 1400 & 910 & 1.15 & 400 & 1.3333 \\
\hline CSF & 398 & 1689 & 311 & 1560 & 0.408 & 0.351 & 1550 & 957 & 1.15 & 399 & 1.3333 \\
\hline CSF & 394 & 1710 & 322 & 1809 & 0.423 & 0.368 & 1690 & 1059 & 1.14 & 394 & 1.3333 \\
\hline ms & 340 & 11750 & 145 & 8320 & 0.779 & 0.557 & 4160 & 2912 & 1.4 & 340 & 1.3334 \\
\hline ms & 336 & 11745 & 1444 & 8310 & 0.773 & 0.534 & 4422 & 3219 & 1.4 & 336 & 1.3439 \\
\hline ms & 245 & 1610 & 110 & 2212 & 0.465 & 0.941 & 1440 & 2276 & 0.495 & 227 & 1.3641 \\
\hline gmatter & 130 & 1601 & 72 & 2209 & 0.464 & 0.938 & 1439 & 361 & 0.491 & 166 & 1.3956 \\
\hline gmatter & 128 & 1597 & 74 & 2206 & 0.463 & 0.934 & 1435 & 351 & 0.489 & 165 & 1.3956 \\
\hline w matter & 95 & 1180 & 70 & 2443 & 0.453 & 0.788 & 1345 & 312 & 0.488 & 148 & 1.4251 \\
\hline edema & 160 & 1231 & 132 & 2216 & 0.443 & 0.776 & 1123 & 325 & 0.467 & 246 & 1.3741 \\
\hline edema & 193 & 1452 & 130 & 2340 & 0.441 & 0.768 & 990 & 312 & 0.445 & 245 & 1.3823 \\
\hline GLIOMA & 90 & 1443 & 127 & 2243 & 0.431 & 0.766 & 989 & 310 & 0.423 & 175 & 1.4331 \\
\hline Gblastma & 108 & 2457 & 154 & 2115 & 0.332 & 0.676 & 900 & 311 & 0.311 & 195 & 1.4512 \\
\hline Gblastma & 128 & 1284 & 131 & 2589 & 0.541 & 0.781 & 1767 & 322 & 0.76651 & 198 & 1.4723 \\
\hline METS & 130 & 1301 & 130 & 2478 & 0.511 & 0.657 & 1011 & 323 & 0.432 & 200 & 1.4831 \\
\hline METS & 152 & 1412 & 132 & 2022 & 0.425 & 0.713 & 1121 & 357 & 0.451 & 224 & 1.4913 \\
\hline
\end{tabular}

\subsection{Testing the SVM to predict the classes of unknown data sets}

Using a few data sets (which were not used to train the SVM) we test the SVM. The data (Table 3) to the SVM is fed and it predicts the classes on its own.

Table 3: Test data set. The trained SVM predicts the classes for 19 unknown data sets.

\begin{tabular}{|c|c|c|c|c|c|c|c|c|c|c|c|}
\hline TISSUE & T2 & CHO & ADC & CR & CHO/NAA & CR/NAA & LIP/LAC & MI & CH/CR & T2peri & RI \\
\hline & & & & & & & & & & & \\
\hline CSF & 400 & 1610 & 300 & 1400 & 0.402 & 0.346 & 1400 & 910 & 1.15 & 400 & 1.3333 \\
\hline CSF & 399 & 1676 & 307 & 1450 & 0.404 & 0.347 & 1489 & 917 & 1.15 & 399 & 1.3333 \\
\hline CSF & 398 & 1689 & 311 & 1560 & 0.408 & 0.351 & 1550 & 957 & 1.15 & 399 & 1.3333 \\
\hline CSF & 395 & 1711 & 322 & 1800 & 0.422 & 0.367 & 1701 & 1056 & 1.14 & 395 & 1.3333 \\
\hline CSF & 394 & 1710 & 322 & 1809 & 0.423 & 0.368 & 1690 & 1059 & 1.14 & 394 & 1.3333 \\
\hline ms & 337 & 11746 & 1445 & 8311 & 0.774 & 0.536 & 4421 & 3220 & 1.4 & 337 & 1.3421 \\
\hline ms & 335 & 11745 & 1443 & 8309 & 0.772 & 0.532 & 4420 & 3216 & 1.4 & 335 & 1.3498 \\
\hline$m s$ & 334 & 11743 & 1443 & 8308 & 0.771 & 0.531 & 4419 & 3214 & 1.4 & 334 & 1.3499 \\
\hline ms & 304 & 5947 & 120 & 5400 & 0.873 & 0.7396 & 6766 & 4294 & 1.1 & 245 & 1.3519 \\
\hline
\end{tabular}

U R L : http://dx.doi.org/10.14738/jbemi.53.4700 
Journal of Biomedical Engineering and Medical Imaging, Volume 5, No 3, June 2018

\begin{tabular}{|c|c|c|c|c|c|c|c|c|c|c|c|}
\hline gmatter & 128 & 1597 & 74 & 2206 & 0.463 & 0.934 & 1435 & 351 & 0.489 & 165 & 1.3956 \\
\hline w matter & 95 & 1180 & 70 & 2443 & 0.453 & 0.788 & 1345 & 312 & 0.488 & 148 & 1.4251 \\
\hline w matter & 93 & 1067 & 89 & 2301 & 0.444 & 0.775 & 1167 & 324 & 0.466 & 169 & 1.4262 \\
\hline edema & 191 & 1451 & 131 & 2356 & 0.441 & 0.778 & 990 & 313 & 0.445 & 245 & 1.3822 \\
\hline edema & 193 & 1452 & 130 & 2340 & 0.441 & 0.768 & 990 & 312 & 0.445 & 245 & 1.3823 \\
\hline GLIOMA & 90 & 1443 & 127 & 2243 & 0.431 & 0.766 & 989 & 310 & 0.423 & 175 & 1.4331 \\
\hline GLIOMA & 107 & 2213 & 155 & 2114 & 0.333 & 0.677 & 901 & 310 & 0.321 & 191 & 1.4456 \\
\hline Gblastma & 108 & 2457 & 154 & 2115 & 0.332 & 0.676 & 900 & 311 & 0.311 & 195 & 1.4512 \\
\hline METS & 129 & 1298 & 130 & 2567 & 0.511 & 0.657 & 1011 & 323 & 0.432 & 200 & 1.4831 \\
\hline METS & 152 & 1412 & 132 & 2022 & 0.425 & 0.713 & 1121 & 357 & 0.451 & 224 & 1.4913 \\
\hline
\end{tabular}

\section{Results}

We ran our trained SVM on a data set of 19 patients. These 19 sets were excluded from the training set for obvious reasons. We had the original diagnoses prior to running the code. The SVM successfully classified each of the 19 data sets accurately. No misclassification was encountered. We retrained the SVM by taking different data sets out of the training set and using them for test purposes, owing to lack of new data. Multiple tests yielded consistently accurate results i.e. the SVM perfectly classified the data with $0 \%$ uncertainty or false classifications. Thus, this Support Vector Machine enabled code correctly diagnoses the seven different types of brain diseases reliably. It also clearly differentiates between normal CSF tissue and lesions/pathological conditions.

Results are recorded in Table 4.

Table 4: Final results comparing the SVM prediction to the actual diagnoses.

\begin{tabular}{|c|c|c|}
\hline ORIGINAL PATIENT DIAGNOSIS & RESULT PREDICTED BY OUR SVM & ACCURACY OF PREDICTION \\
\hline 'CSF' & 'CSF' & Accurate Classification \\
\hline 'CSF' & 'CSF' & Accurate Classification \\
\hline 'ms' & 'ms' & Accurate Classification \\
\hline 'ms' & 'ms' & Accurate Classification \\
\hline 'gmatter' & 'gmatter' & Accurate Classification \\
\hline 'gmatter' & 'gmatter' & Accurate Classification \\
\hline 'w matter' & 'w matter' & Accurate Classification \\
\hline 'w matter' & 'w matter' & Accurate Classification \\
\hline 'edema' & 'edema' & Accurate Classification \\
\hline 'edema' & 'edema' & Accurate Classification \\
\hline 'GLIOMA' & 'GLIOMA' & Accurate Classification \\
\hline 'GLIOMA' & 'GLIOMA' & Accurate Classification \\
\hline 'Gblastma' & 'Gblastma' & Accurate Classification \\
\hline 'Gblastma' & 'Gblastma' & Accurate Classification \\
\hline 'Gblastma' & 'Gblastma' & Accurate Classification \\
\hline 'METS' & 'METS' & Accurate Classification \\
\hline 'METS' & 'METS' & Accurate Classification \\
'METS' & 'METS' & Accurate Classification \\
\hline 'METS' & 'METS' & Accurate Classification \\
\hline
\end{tabular}

\section{Conclusion}

It can be concluded that the use of Support Vector Machines and other tools of Machine Learning [8] can be employed to achieve extremely reliable and accurate diagnostic results in cases where conventional imaging techniques offer less clarity, and where biopsies [9] carry potential risks to the 
Sparsh Jain, Tapan K Biswas, Rajib Bandyopadhyay; Diagnosis of Brain Lesions, Glioma, Multiple-Sclerosis and Metastases from MRI: An efficient classifier-aided method using Refractive Index as a surrogate Biological Marker. Journal of Biomedical Engineering and Medical Imaging, Volume 5, No 3, June (2018), pp -19-26

patient. An added advantage of using SVM for this purpose is that it guarantees reproducibility and repeatability i.e. the accuracy levels are guaranteed to remain fairly constant over multiple runs and long periods of time. Along with the custom-developed colour palette [10] using refractive index, it also provides a much sharper contrast image of the brain. We here note that the accuracy is limited only by the size of the data set we use for training the SVM initially. Owing to our present setup, we are unable to record new data on a rolling basis. A further step towards making it more reliable and robust would include obtaining new data in large amounts, and incorporating these data points into the training set. Different classification algorithms can further be integrated with this to achieve even better and conclusive results in all cases. It is safe to declare that Support Vector Machines will find their use in dayto-day radiological and imaging methods for common use due to their superior accuracy, fail-proof safety fallbacks and evolving classification algorithms. This in turn will slowly reduce the need to perform invasive biopsies and multiple tests, as well as make diagnoses less dependent on the human radiologist/ practitioner who might be prone to making errors [11].

\section{REFERENCES}

[1] Schwab, K.E., Gailloud P, Wyse G, Tamargo R.J., Limitations of magnetic resonance imaging and magnetic resonance angiography in the diagnosis of intracranial aneurysms. Neurosurgery, Volume 63, Issue 1, 1 July 2008

[2] Support Vector Machines - http://www.statsoft.com/Textbook/Support-Vector-Machines

[3] Mingxia Liu, Daoqiang Zhang, Joint Binary Classifier Learning for ECOC-Based Multi-Class Classification. IEEE Transactions on Pattern Analysis and Machine Intelligence ( Volume: 38, Issue: 11, Nov. 12016 )

[4] Pardalos P.M. (2008) Hyperplane Arrangements in Optimization. In: Floudas C., Pardalos P. (eds) Encyclopedia of Optimization. Springer, Boston, MA

[5] Tapan K Biswas, R Bandyopadhyay, A Dutta, Validating The Discriminating Efficacy Of MR T2 Relaxation Value Of Different Brain Lesions And Comparison With Other Differentiating Factors: Use Of Artificial Neural Network And Principal Component Analysis. The Internet Journal of Radiology (Volume 20, No. 1)

[6] Radiopedia Introduction to MR Spectroscopy, https://radiopaedia.org/articles/mr-spectroscopy-1.

[7] Mathworks Guide, Support Vector Machines for Binary Classification.

[8] Machine Learning, Wikipedia, https://en.wikipedia.org/wiki/Machine_learning.

[9] Nor M., Noor Rahman, J. Adnan, Intracranial Bleed Post Stereotactic Biopsy: Lessons Learned, The Internet Journal of Neurosurgery (Volume 8, Number 1)

[10] Tapan K Biswas, S R Choudhury, A Ganguly, R Bandyopadhyay, A Dutta, Refractive Index As Surrogate Biological Marker Of Tumefactive And Other Form Of Multiple Sclerosis And Its Superiority Over Other Methods. The Internet Journal of Radiology (Volume 19, Number 1).

[11] Blake A. Johnson, Avoiding diagnostic pitfalls in neuroimaging. Applied Radiology-The Journal of Practical Medical Imaging and Management 2016;45(3):24-29. March 02, 2016 
Journal of Biomedical Engineering and Medical Imaging, Volume 5, No 3, June 2018

[12] Wikipedia, Nuclear Magnetic Resonance Spectroscopy, https://en.wikipedia.org/wiki/Nuclear_magnetic_resonance_spectroscopy

[13] Kasai, M., Yasuda, Y., Mizoguchi, H., Soga, K., Kaneko, K., \& Takemura, H. (2017). In vivo tumor wavelength band selection using Hierarchical clustering and PCA with NIR-Hyperspectral Data. Journal of Biomedical Engineering and Medical Imaging, 4(1), 01.

[14] Limam, O. (2016). MRI Segmentation based on Multiobjective Fuzzy Clustering. Journal of Biomedical Engineering and Medical Imaging, 3(2), 07.

[15] Abd El kader, I., Zhang, S., \& Xu, G. (2017). Improved Fuzzy C-Means Algorithm for Brain Tumor Identification Analysis Using Magnetic Resonance Brain Images. Journal of Biomedical Engineering and Medical Imaging, 4(3), 15. 